\title{
A Promoção de Novos Produtos de Tabaco nas Redes Sociais à Luz da Pandemia
}

doi: https://doi.org/10.32635/2176-9745.RBC.2020v66nTemaAtual.1108

\author{
Promoting New Tobacco Products in Social Media in Pandemic Times \\ La Promoción de Nuevos Productos de Tabaco en las Redes Sociales a la Luz de la Pandemia
}

Mariana Coutinho Marques de Pinho'; Maria Paula Russo Riva²; Laura de Souza Cury33; Mônica Andreis

RESUMO

Introduçáo: Sofisticadas estratégias de marketing têm sido utilizadas para atrair o público jovem para o consumo de produtos fumígenos e promover a aceitação social do tabagismo. No Brasil, a venda e publicidade de dispositivos eletrônicos para fumar (DEF) é proibida desde 2009, mas foram detectadas iniciativas para promover esses produtos nas redes sociais. Objetivo: Registrar e analisar açôes de marketing de produtos de tabaco nas redes sociais durante a pandemia da doença pelo coronavírus 2019 (coronavirus disease 2019 - Covid-19). Método: Por meio de uma abordagem qualitativa, este estudo analisou a utilizaçáo da rede social Instagram por usuários e comerciantes de DEF, especialmente o uso de stickers criados pelo aplicativo no período da pandemia de Covid-19. Resultados: O adesivo do Instagram foi usado indevidamente e possibilitou a propaganda e a promoção de DEF em rede social por parte de seus usuários, apesar da proibição das normas brasileiras. Conclusáo: A rede social apresenta alto grau de engajamento e tem 33\% dos usuários em idade entre 13 e 24 anos, faixa etária alvo das estratégias de marketing da indústria do tabaco e comerciantes de DEF. Além da responsabilidade de quem realizou a postagem, há que se considerar a corresponsabilidade do Instagram ao não coibir esse tipo de iniciativa.

Palavras-chave: Tabagismo; Sistemas Eletrônicos de Liberaçáo de Nicotina; Produtos do Tabaco; Publicidade de Produtos Derivados do Tabaco.

\section{ABSTRACT}

Introduction: Sophisticated marketing strategies are used to attract young consumers to smoking and promote social acceptance of tobacco use. In Brazil, advertising and sales of electronic smoking devices (ESD) are banned since 2009, but initiatives have been detected recently to promote these products in the social media. Objective: To register and analyze marketing strategies for tobacco products on social media during the pandemic of coronavirus disease 2019 (COVID-19). Method: Through a qualitative approach, this study analyzed the use of the social network Instagram by ESD users and sellers, especially stickers created by the applicative during the COVID-19 pandemic period. Results: The Instagram sticker was used improperly and favored the advertising and promotion of ESD in the social network by its users, despite the prohibition by the Brazilian legislation. Conclusion: The social network has a high degree of allegiance, $33 \%$ of its users are aged between 13 and 24 years old, an age group targeted by the tobacco industry and sellers of ESD marketing strategies. In addition to holding who made the post accountable, Instagram's co-responsibility must be considered for upholding this type of initiative.

Key word: Tobacco Use Disorder; Electronic Nicotine Delivery Systems; Tobacco Products; Tobacco-Derived Products Publicity.

\section{RESUMEN}

Introducción: Se han utilizado sofisticadas estrategias de marketing para atraer a los jóvenes a fumar y promover la aceptación social del tabaquismo. En Brasil, la prohibición de la publicidad de dispositivos electrónicos para fumar (DEF) se contempla legalmente desde 2009, sin embargo, se detectaron esfuerzos para promocionar estos productos en las redes sociales Objetivo: Registrar y analizar acciones de marketing de productos del tabaco en las redes sociales durante la pandemia del coronavirus 2019 (coronavirus disease 2019 - Covid-19). Método: Utilizando un enfoque cualitativo, este estudio analizó el uso de la red social Instagram por parte de los usuarios y vendedores de DEF, especialmente el uso de stickers creadas por la aplicación durante el período pandémico de la enfermedad por Covid-19. Resultados: El adhesivo de Instagram se usó de manera ilegal e hizo posible la publicidad y promoción de DEF en una red social, a pesar de la prohibición de la normativa brasileńa. Conclusión: La red social tiene un alto grado de participación de jóvenes: el 33\% de los usuarios tienen edades comprendidas entre 13 y 24 años, grupo de edad blanco de las estrategias de marketing de la industria tabacalera y comerciantes de DEF. Además de la responsabilidad de la persona que lo publicó, se debe considerar la corresponsabilidad de Instagram, ya que no actúa en contra de este tipo de iniciativa.

Palavras clave: Tabaquismo; Sistemas Electrónicos de Liberación de Nicotina; Productos de Tabaco; Publicidad de Productos Derivados del Tabaco.

1'Aliança de Controle do Tabagismo (ACT) Promoção da Saúde. Mestranda em Saúde Pública na Escola Nacional de Saúde Pública da Fundação Oswaldo Cruz (Ensp/ Fiocruz). Rio de Janeiro (RJ) Brasil. Orcid iD: https://orcid.org/0000-0002-8372-9272

${ }^{2}$ ACT Promoção da Saúde. Mestre em Direitos Humanos Internacionais pela Queens Mary University. Londres, Inglaterra. Orcid iD: https://orcid.org/0000-0003-2458-9975 ${ }^{3}$ ACT Promoção da Saúde. Doutoranda em História Social na Pontifícia Universidade Católica de São Paulo (PUC-SP). São Paulo (SP), Brasil. Orcid iD: https://orcid. or/0000-0002-6325-4599

${ }^{4}$ ACT Promoção da Saúde. Mestre em Psicologia Clínica pela Universidade de São Paulo (USP). São Paulo (SP), Brasil. Orcid iD: https://orcid.org/0000-0002-9514-0473 Endereço para correspondência: Mariana Coutinho Marques de Pinho. Avenida Nossa Senhora de Copacabana, 330, sala 1107 - Copacabana. Rio de Janeiro (RJ), Brasil. CEP 22020-001. E-mail: mariana.pinho@actbr.or.br 


\section{INTRODUÇÃO}

Esta comunicação breve pretende abordar o tema da publicidade de dispositivos eletrônicos para fumar (DEF) nas redes sociais, especialmente a partir da análise de uma iniciativa adotada durante a pandemia da doença pelo coronavírus 2019 (coronavirus disease 2019 - Covid-19), na rede social on-line Instagram.

O tabagismo, fator de risco para câncer, diabetes, doenças pulmonares e cardiovasculares, mata mais de oito milhôes de pessoas por ano no mundo ${ }^{1}$. Essa pandemia levou a Organização Mundial da Saúde (OMS) a aprovar a Convenção-Quadro para Controle do Tabaco (CQCT) em 2003, primeiro tratado de saúde pública, que conta hoje com a ratificação da União Europeia e 181 países, incluindo o Brasil ${ }^{2}$.

O tabagismo é uma doença caracterizada pela dependência, mas também comumente referido como uma doença transmissível cujo vetor é a propaganda ${ }^{3}$. Isto porque a indústria do tabaco (IT) utiliza sofisticadas estratégias de marketing para atrair consumidores e gerar percepçáo social de que fumar seria normal e aceitável. A publicidade visa a associar o uso de tabaco com valores e comportamentos almejados pelos jovens, como popularidade, independência, glamour e apelo sexual, e inovação, minimizando (quando não negligenciando) os riscos à saúde.

Apesar de as fabricantes afirmarem que não buscam promover seus produtos para o público jovem, documentos internos da IT mostram de forma clara que este é seu público-alvo. O CEO da RJ Reynolds, por exemplo, declarou em 1973 que "[s]e a companhia quiser sobreviver e prosperar no longo prazo, devemos conseguir uma fatia de mercado jovem" ". Revisão sistemática realizada por Lovato et al. ${ }^{5}$ com mais de 29 mil adolescentes concluiu que a propaganda de tabaco aumenta a probabilidade de jovens começarem a fumar.

No Brasil, a restrição à publicidade de tabaco tem previsão legal desde 1996, e foi recentemente atualizada e regulamentada pela Agência Nacional de Vigilância Sanitária (Anvisa) em 2011 e 2018, respectivamente. A Resolução da Diretoria Colegiada da Anvisa (RDC/ Anvisa) no. 213/2018 ${ }^{6}$ define o que é propaganda de produtos fumígenos e dispóe ser ilegal qualquer forma de publicidade que não se atenha à exposição da embalagem no ponto de venda. Tais medidas encontram consonância com o estabelecido pelo artigo 13 da CQCT, que se refere à publicidade, promoção e patrocínio de tabaco.

Visando a manter lucros bilionários, a IT inova constantemente. Exemplo disso são os cigarros eletrônicos e os produtos de tabaco aquecido - denominados DEF - heterogêneo, grupo de produtos alimentados à bateria que, em sua esmagadora maioria, contêm nicotina, na forma líquida ou da folha de tabaco.
Alegam os fabricantes que os DEF seriam produtos com risco reduzido e voltados para tabagistas que não querem/conseguem parar de fumar. No entanto, a experiência internacional mostra que, em países onde esses produtos foram introduzidos, houve um aumento exponencial do seu uso entre jovens, a ponto de, nos Estados Unidos, a Food and Drug Administration ter declarado se tratar de uma epidemia, com um aumento de $135 \%$ no uso entre 2017-20197.

No Brasil, a RDC/Anvisa no. 46/2009 ${ }^{8}$ veda a comercialização, importação e propaganda de quaisquer DEF, especialmente aqueles que alegam substituir outros produtos fumígenos ou que objetivem caracterizar-se como alternativa no tratamento do tabagismo.

Os aspectos relativos à propaganda de produtos de tabaco, incluindo os DEF, se tornam ainda mais importantes no atual contexto da pandemia da Covid-19, tendo em vista que seus usuários têm mais chances de desenvolver doenças pulmonares e vasculares, sistemas esses igualmente prejudicados nos infectados pela síndrome respiratória aguda grave do coronavírus 2 (severe acute respiratory syndrome coronavirus 2 - Sars-CoV-2 $)^{9-11}$.

De forma ilegal, açóes sorrateiras de marketing nas redes sociais vêm sendo desenvolvidas pelos fabricantes e comerciantes de $\mathrm{DEF}^{12}$. Os jovens são assíduos frequentadores dos meios digitais e essas postagens podem torná-los suscetíveis à experimentação e consumo desses $\operatorname{produtos}^{13}$.

Desse modo, o registro e a análise desse tipo de atividade, especialmente durante a pandemia, são relevantes para a saúde pública, pois têm potencial de impactar na iniciação por jovens de produtos nocivos e que causam dependência.

\section{MÉTODO}

Por meio de uma abordagem qualitativa, este estudo analisou a utilização da rede social Instagram por usuários e comerciantes de DEF, especialmente o uso de stickers criados pelo aplicativo no período da pandemia de Covid-19. As pesquisadoras capturaram imagens publicadas no Instagram, no Stories de perfis públicos brasileiros, e analisaram seus potenciais prejuízos para Política Nacional de Controle do Tabaco no Brasil.

\section{RESULTADOS}

Desde a adoção do distanciamento físico, o Instagram desenvolveu stickers (adesivos) para serem usados em postagens dos Stories. O adesivo "Em casa" refere-se à orientação de distanciamento físico como medida para impedir maior transmissão do Sars-CoV-2. O sticker 
"Agradeço" homenageia profissionais de saúde que estáo na linha de frente.

Em maio, a plataforma criou o adesivo "Apoie as pequenas empresas", como forma de estimular usuários a adquirirem produtos e serviços de fornecedores locais, que são mais impactados economicamente pelas regras do bloqueio comercial (Figura 1) ${ }^{14}$.

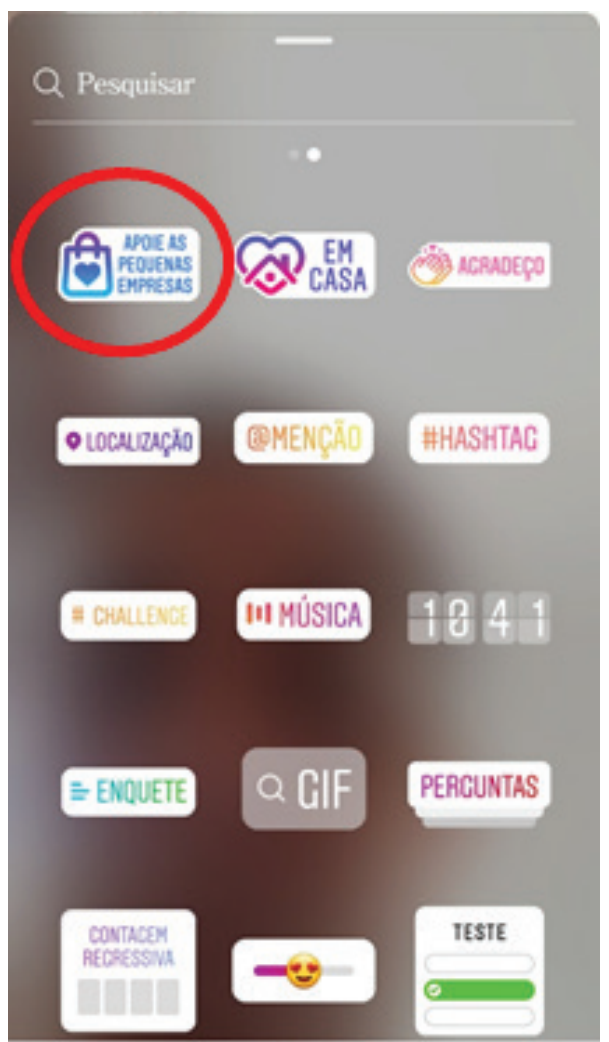

Figura 1. Lista de recursos do Stories do Instagram Nota: O sticker "Apoie as pequenas empresas" aparece em destaque como o primeiro recurso a ser aplicado à imagem a ser compartilhada.
A ação pode parecer louvável, mas pouco dias depois de seu lançamento, perfis de vapers (usuários de DEF) e de revendedores desses produtos passaram a adotar o sticker para promover produtos e fornecedores. Os registros mostram e-juices ou e-liquids (substâncias utilizadas nos aparelhos para conferir sabor e aroma), peças para vapes, aparelhos de vapear e/ou qualquer outra imagem relacionada a vaping.

O adesivo é adicionado na imagem original, identificando um segundo usuário, provável fornecedor do produto promovido. Cada postagem permanece visível no Stories do primeiro usuário por 24 horas. Por sua vez, o usuário-fornecedor é notificado e pode compartilhá-la como forma de agradecimento e, certamente, para promover seus negócios, prolongando assim a ação de marketing pelo tempo que desejar (Figura 2).

Ao tentarem denunciar essa propaganda de DEF, as pesquisadoras receberam mensagens automáticas da plataforma informando que náo seria possível analisar a denúncia em razão da sobrecarga imposta pela Covid-19; somente "conteúdo com mais potencial de ser prejudicial" seria analisado (Figura 3). Apesar de, no final de 2019, o Instagram ter alterado sua política de uso "para incluir regras mais claras para a divulgaçáo paga de determinados bens e serviços, como vaping, tabaco (...) [, proibindo] conteúdos de marca que promovam vaping, produtos derivados do tabaco", usuários da plataforma não conseguem, no momento, denunciar a propaganda de $\mathrm{DEF}^{15}$.

\section{DISCUSSÃO}

O sticker do Instagram foi usado indevidamente e possibilitou a propaganda e a promoçấo de DEF em rede
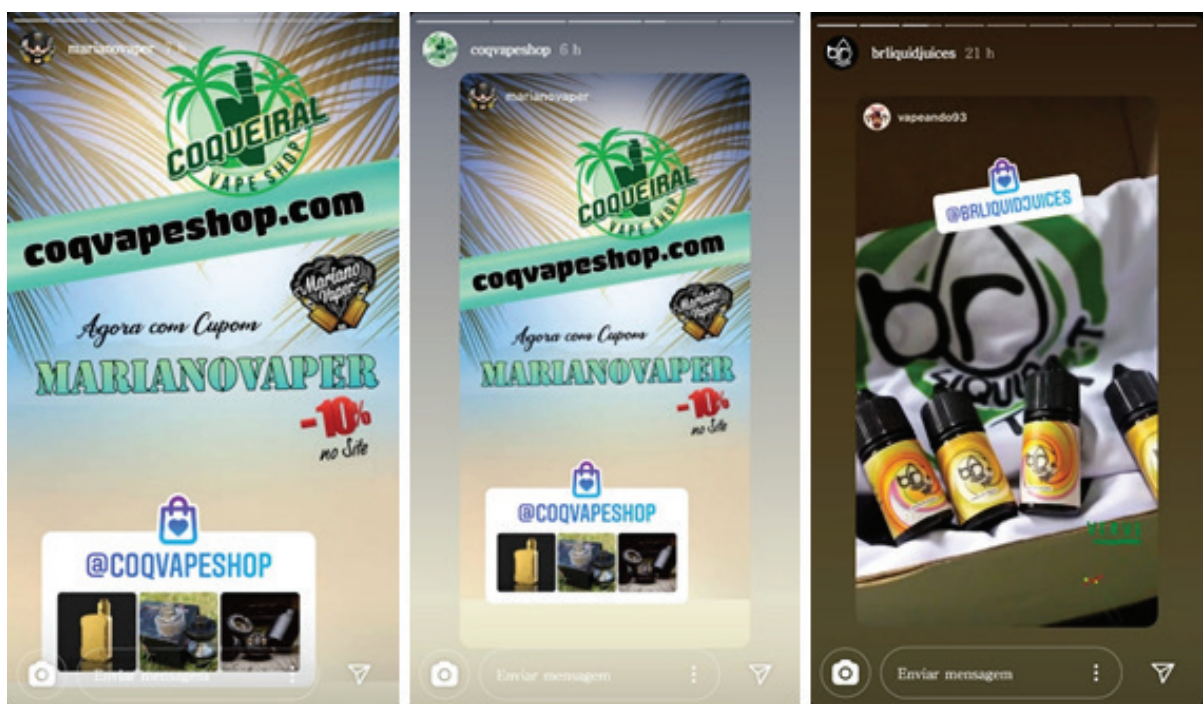

Figura 2. Postagens originais e compartilhadas com o adesivo "Apoie as pequenas empresas"

Nota: À esquerda, a postagem original feita por @marianovaper. Na imagem central, a postagem compartilhada feita por usuário-fornecedor (@coqvapeshop). À direita, o sticker no seu formato reduzido em imagem compartilhada por usuário-fornecedor (@brliquidjuices). 


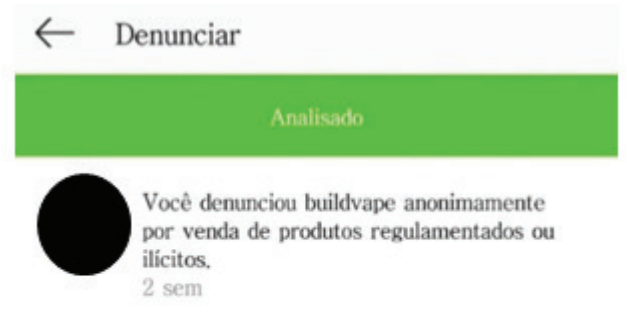

(O) Não podemos priorizar todas as denúncias

Temos menos pessoas disponiveis para analisar as denúncias devido à pandemia do coronavírus (COVID-19). Por isso, só estamos conseguindo analisar o conteúdo com mais potencial de ser prejudicial. Se não quer ver buildvape no Instagram. você pode deixar de seguir, silenciar ou bloquear essa pessoa para ocultar as publicações e comentários dela no seu feed.

Denúncias como a sua são importantes para ajudar a tornar o Instagram um lugar seguro e acolhedor para todos.

2 sem

\section{Mais opcres}

Figura 3. Resposta automática do Instagram à denúncia de conteúdo de propaganda de DEF

social por parte de seus usuários, apesar de clara proibição da Lei Federal e RDC/Anvisa.

Conclusóes feitas por Ratneswaran et al. ${ }^{16}$ apontam que a propaganda de cigarro eletrônico estimula o uso desses produtos e de cigarro convencional entre jovens fumantes e não fumantes. Análise feita pela Forrester aponta o Instagram como a rede social que mais promove o engajamento dos consumidores com marcas, a uma taxa de $4,21 \%{ }^{17}$. Esse elevado grau de engajamento é especialmente preocupante, tendo em vista que $31,4 \%$ dos usuários do Instagram têm idade entre 13 e 24 anos $^{18}$, faixa etária alvo das estratégias de marketing da IT e fabricantes de DEF.

$\mathrm{O}$ marketing digital cria um vácuo de responsabilidade, além de incertezas regulatórias. A formulação, aprovação e implementação de políticas de controle podem ser um processo moroso, mas urge que adaptaçóes sejam feitas e constantemente revisadas, conforme a própria evolução da tecnologia e das mídias, e que a regulação seja monitorada e implementada.

Além da responsabilidade de quem realizou a postagem, há que se considerar a corresponsabilidade do Instagram ao não coibir esse tipo de iniciativa. Apenas incluir em sua Política que não mais permite propaganda de DEF não tem sido suficiente. Isso se agrava no contexto atual, considerando que a plataforma não atendeu às denúncias feitas.
Assim como foi identificada essa iniciativa, é importante monitorar e expor outras atividades de promoção indevida de produtos fumígenos, seja em mídias sociais ou em outros veículos, a fim de zelar pelo cumprimento dos dispositivos legais vigentes e pela prevenção do tabagismo por jovens.

Complementarmente, desde o início da pandemia da Covid-19, o setor privado vem afirmando seu comprometimento ao ajudar na crise sanitária e econômica resultante. Embora iniciativas de auxílio sejam bem-vindas, o momento exige cautela. Alguns setores enxergam, nesse contexto, "oportunidades" a serem abordadas usando valores de responsabilidade social corporativa. No entanto, tais ações devem ser compreendidas como ações de marketing ${ }^{19}$.

\section{CONCLUSÃO}

A Política Nacional Brasileira de Controle do Tabaco prevê uma série de medidas de prevenção à iniciação ao tabagismo e vem colhendo resultados positivos na redução do percentual de fumantes. No entanto, açóes que impeçam seu avanço, como a propaganda em rede social, podem resultar em jovens experimentando e se tornando adultos fumantes que perderão precocemente suas vidas.

É inaceitável que, além de desrespeitarem a legislação vigente, usuários de redes sociais utilizem indevidamente a iniciativa criada no sentido de favorecer comerciantes locais durante período da pandemia de Covid-19. E é também inaceitável que a rede social Instagram se omita diante dessa publicidade ilegal.

Estamos testemunhando náo apenas a promoção ilegal desses produtos, mas também a omissão de uma das plataformas digitais mais acessadas na atualidade. $\mathrm{O}$ cuidado às pessoas e à saúde inclui a responsabilidade diante da tentativa de atrair consumidores, notadamente jovens, à experimentação e ao uso regular dos DEF.

\section{CONTRIBUIÇÕES}

Todos os autores contribuíram na concepção e/ou no planejamento do estudo; na obtenção, na análise e na interpretação dos dados; assim como na redaçáo e revisão crítica; e aprovaram a versão final a ser publicada.

\section{AGRADECIMENTOS}

À equipe do Centro de Estudos sobre Tabaco e Saúde da Escola Nacional de Saúde Pública Sérgio Arouca da Fundação Oswaldo Cruz (Cetab/Ensp/Fiocruz), que sedia o Observatório sobre as Estratégias da Indústria do Tabaco e disponibiliza relevante repositório de informaçôes a esse respeito. 


\section{DECLARAÇÃO DE CONFLITO DE INTERESSES}

Nada a declarar.

\section{FONTES DE FINANCIAMENTO}

O monitoramento das estratégias da IT é realizado regularmente pela ACT Promoção da Saúde e com apoio financeiro parcial da organização não governamental Campaign for Tobacco-Free Kids e Bloomberg Philantropies.

\section{REFERÊNCIAS}

1. World Health Organization [Internet]. Geneva: WHO; c2020. Tobacco: key facts; 2020 May 27 [cited 2020 Jan 16]. Available from: https://www.who.int/en/newsroom/fact-sheets/detail/tobacco

2. WHO Framework Convention on Tobacco Control [Internet]. Geneva: WHO; c2020. Parties to the WHO Framework Convention on Tobacco Control; [2005] [cited 2020 June 19]. Available from: http://www.who. int/fctc/cop/en

3. Szklo AS, Mendes FL, Cavalcante TM, et al. Interferência da indústria do tabaco no Brasil: a necessidade do ajuste de contas. Rev Bras Cancerol. 2020;66(2):e11878. doi: https://doi.org/10.32635/2176-9745. RBC.2020v66n2.878

4. Aliança de Controle do Tabagismo. O veredicto final: trechos do processo Estados Unidos X Philip Morris. São Paulo: ACTbr; OPAS; 2008.

5. Lovato C, Watts A, Stead LF. Impact of tobacco advertising and promotion on increasing adolescent smoking behaviours. Cochrane Database Syst Rev. 2011;2011(10):CD003439. doi: https://doi. org/10.1002/14651858.CD003439.pub2

6. Agência Nacional de Vigilância Sanitária (BR). Resolução da Diretoria Colegiada no 213, de 23 de janeiro de 2018. Dispóe sobre a exposição à venda e a comercialização de produtos fumígenos derivados do tabaco [Internet]. Diário Oficial da União. 2018 jan 26; Seção 1:31 [acesso 2020 ago 18]. Disponível em: http://portal. anvisa.gov.br/documents/10181/3219394/\%281\%29 RDC_213_2018_COMP.pdf/fb3c20d8-1b97-47dfafdb-96e350bce38f

7. Bach L. JUUL and youth: rising e-cigarette popularity. Campaign for Tobacco-Free Kids; 2019 Dec 12.

8. Agência Nacional de Vigilância Sanitária (BR). Resolução da Diretoria Colegiada no 46, de 28 de agosto de 2009. Proíbe a comercialização, a importação e a propaganda de quaisquer dispositivos eletrônicos para fumar, conhecidos como cigarro eletrônico [Internet]. Diário Oficial da União. 2009 ago 31; Seção 1:45 [acesso 2020 ago 18]. Disponível em: http://portal.anvisa.gov.br/ documents/10181/2718376/RDC_46_2009_COMP. pdf/2148a322-03ad-42c3-b5ba-718243bd1919
9. World Health Organization [Internet]. Geneva: WHO; c2020. WHO statement: tobacco use and COVID-19; 2020 Mayo 11 [cited 2020 June 16]. Available from: https://www.who.int/news-room/detail/11-05-2020who-statement-tobacco-use-and-covid-19.

10. Szklo AS. Associação entre fumar e progressão para complicaçóes respiratórias graves em pacientes com Covid-19. Rev Bras Cancerol. 2020;66(2):e03974. doi: https://doi.org/10.32635/2176-9745. RBC.2020v66n2.974

11. Campaign for Tobacco-Free Kids [Internet]. Washington, D.C.: Campaign for Tobacco-Free Kids; C2017. Tabagismo, uso de cigarro eletrônico e COVID-19: evidências emergentes; 2020 maio [acesso 2020 Aug 18]. Disponível em: http://www.actbr.org.br/uploads/ arquivos/200604-Smoking-Vaping-and-COVID19_ Evidence_PT\%281\%29.pdf

12. Juliana. Denúncia: marketing ilegal de cigarros em mídias sociais. 2018 nov 9 [acesso 2020 jun 19]. In: Blog ACT Promoção da Saúde [Internet]. Rio de Janeiro; São Paulo: ACT Promoção da Saúde [data desconhecida]. Disponível em: http://blog.actbr.org.br/controle-dotabagismo/denuncia-marketing-ilegal/1900

13. Echosis [Internet]. Lages, SC: Echosis; [data desconhecida]. Instagram e Snapchat se firmam como as redes mais usadas pelos jovens; 2018 nov 27 [acesso 2020 jun 19]. Disponível em: https://www.echosis.com. br/instagram-e-snapchat-se-firmam-como-as-redes-maisusadas-pelos-jovens/

14. Techtudo [Internet]. Rio de Janeiro: Globo Comunicação e Participaçôes; c200-2020. Beggiora H. 'Apoie pequenas empresas': como usar o novo sticker do Instagram; 2020 maio 11 [acesso 2020 jun 16]. Disponível em: https:// www.techtudo.com.br/dicas-e-tutoriais/2020/05/ apoie-pequenas-empresas-como-usar-o-novo-stickerdo-instagram.ghtml

15. Instragran Empresas. [blog]. São Francisco, CA: Instagran. c2020 - . Ajudando criadores de conteúdo a transformar suas paixóes em meio de vida; 2019 dez 18 [acesso 2020 ago 18]. Disponível em: https://business. instagram.com/blog/helping-creators-turn-their-passioninto-a-living/

16. Ratneswaran C, Steier J, Reed K, et al. Electronic cigarette advertising impacts adversely on smoking behaviour within a london student cohort: a cross-sectional structured survey. Lung. 2019;197(5):533-40. doi: https://doi.org/10.1007/s00408-019-00262-z

17. Aragão FBP, Farias FG, Mota MO, et al. Curtiu, comentou, comprou. A mídia social digital Instagram e o consumo. Rev Ciênc Admin. 2016;22(1):130-61. doi: https://doi.org/10.5020/2318-0722.2016.v22n1p130

18. Statista [Internet]. Nova York: Statista; [date unknown]. Distribution of Instagram users in Brazil as of August 2020, by age group; 2020 [cited 2020 Aug 18]. Available 
from: https://www.statista.com/statistics/866268/ instagram-user-share-brazil-age/, acesso em:

19. Bialous SA, Freeman B. Article 5.3 of the WHO Framework Convention on Tobacco Control \& the COVID19 pandemic. 2020 May 10 [cited 2020 June 16]. In: BMJ Blogs [Internet]. London: BMJ Publishing Group. C2020. Available from: https://blogs.bmj.com/ tc/2020/05/10/article-5-3-of-the-who-frameworkconvention-on-tobacco-control-the-covid19-pandemic

Recebido em 23/6/2020

Aprovado em 17/7/2020 